\title{
Ray and wave dynamical properties of a spiral-shaped dielectric microcavity
}

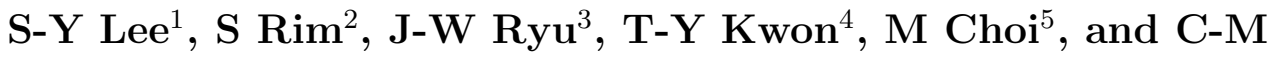 \\ $\mathrm{Kim}^{2}$ \\ ${ }^{1}$ Department of Physics, Seoul National University, Seoul 151-747, Republic of Korea \\ E-mail: pmzsyl@phya.snu.ac.kr \\ ${ }^{2}$ National Creative Research Initiative Center for Quantum Chaos Applications, \\ Sogang University, Seoul 121-742, Republic of Korea \\ 3 Department of Physics, Pusan National University, Busan 609-735, Republic of \\ Korea \\ ${ }^{4}$ Max-Planck Institute for the Physics of Complex Systems, Noethnitzer Str. 38, \\ D-01187, Dresden, Germany \\ ${ }^{5}$ Department of Nonlinear Science, ATR Wave Engineering Laboratories, 2-2-2 \\ Hikaridai, Seika-cho Soraku-gun Kyoto 619-0288, Japan \\ E-mail: chmkim@sogang.ac.kr
}

\begin{abstract}
We investigate ray dynamical properties and resonance patterns of a spiral-shaped dielectric microcavity in which quasiscarred resonances can be supported. The ray dynamical properties of this open system can be characterized by the steady probability distribution which contains informations of the dynamics and the openness of the chaotic microcavity. It is shown that the quasiscarring phenomenon can be understood by considering the unique properties of wave propagation at the dielectric boundary. The bouncing positions of the quasiscarred resonances are explained through a semiclassical quantization condition with Maslov indices. We also show qualitative agreements between the ray dynamical distributions and the wave dynamical distributions obtained from the average over resonance modes.
\end{abstract}

PACS numbers: 05.45.Mt, 42.55.Sa 


\section{Introduction}

Microcavity (micro-disk, -cylinder, or -droplet) lasers have been much studied recently as a new idea that might replace purely electronic large-scale integrated circuits by photonic or optoelectrical circuits [1]. Initial interest was concentrated on generating high $Q$ resonances. The high $Q$ modes of microcavity lasers are based on so-called whispering gallery modes (WGMs) in which rays circulate inside the boundary of circular microcavity and are completely confined by total internal reflection. The advantage of these modes is the small losses only due to evanescent leakage (tunneling) and scattering from the surface roughness. Although the microcavity lasers based on WGM exhibit a high performance of low-threshold current density and low noise, they suffer from small output powers and isotropic emissions because of the high-reflectivity and the rotational symmetry.

In this respect, however, asymmetric resonant cavities (ARC) are more promising concept to achieve high power and directional emissions. Gmachl et al. [2] have reported that a completely different type of resonance compared to WGM, so-called bow-tie resonance, emerges and is responsible for highly directional and high-power emission for "flattened" quadrupole shape cavity laser. Since then, many different boundary shapes smoothly deformed from circle, e.g., ellipse, quadrupole, hexadecapole, and etc., are studied so far. (Note that most of these ARCs are not "truely" asymmetric because they have reflection symmetries for major and minor axes of cavities.) Their farfield emission patterns show reproducible anisotropic patterns and dramatic sensitivity depending on the boundary shapes [3]. Most recently, Chern et al. reported that even unidirectional emissions are possible from a truely asymmetric microcavity, a spiral-shaped microcavity [4]. Besides the photonics applications such as optical computing and networking, study of deformed microcavities can also provides invaluable pedagogical insight into cavity quantum electrodynamics [5, 6], chaotic transport phenomena [7, 8, 9], and even the theory of quantum chaos [1].

In general, statistics of quantum mechanical eigenvalues and eigenfunctions of classically chaotic systems can be well described by random matrix theory (RMT) [10]. From the viewpoint of RMT the existence of scarred eigenfunctions [11], which show enhanced probability amplitude along an unstable periodic orbit, cannot be justified. The scar phenomenon would, therefore, be regarded as the first correction on RMT containing specific information of the chaotic system concerned [12, 13, 14, 15, 16]. The scar phenomenon has been most intriguing aspect of quantum manifestations of classical chaos. Since the seminal finding of scarred eigenfunctions in a chaotic stadium billiard has been reported by Heller [11], many authors have studied this abnormal phenomenon both theoretically and numerically to understand the impact of classical periodic orbits in various chaotic systems [11, 17, 18]. Experimental investigations of quantum chaos have been performed in various contexts such as microwave cavity, quantum dots, surface waves, etc. $[19,20,21,22,23]$ since the original microwave experiment had been performed in 1990 by Stöckmann and Stein [24]. Scarred modes 
were first noted by Sridhar and Heller [25] in microwave experiment of Sinai-billiard.

The recent growing interests in microcavity lasers naturally bring up again the topic of observing evidences of scarred modes in optical systems. There are series of reports on observation of scarred lasing modes in dielectric microcavities of various boundary shapes [26, 27, 28, 29]. In these papers the authors have identified the scarred lasing modes by matching the directionality of lasing emission (far-field emission pattern) and possible unstable periodic orbit in the chaotic microcavities. They took it for granted that the scar theory of chaotic billiards would be applied to the resonances of microcavities without any modification. However, basically dielectric microcavities have quite different classical dynamics compared with that of billiard systems. The major differences are twofold. First, it is an open system, i.e., the energy confined in cavity is a decreasing function of time and the energy loss corresponds to the escaping energy by emission. Second, the refractive index $n$ plays a crucial role in characterizing ray dynamics in the microcavities, e.g., it determines the critical angle of total internal reflection, $\theta_{c}=\arcsin (1 / n)$, and $\theta_{c}$ is, in turn, closely related to directionality of rays on the boundary. We note that these inherent characteristics of dielectric cavities can give rise to important and enormous differences in resonance patterns. For example, the spatial splitting in the type I scarred resonance patterns and the unique far field distribution of the type II scarred resonances [30] can be understood based on characteristics of dielectric cavities. More striking example should be the existence of quasiscarred resonances in dielectric cavities [31].

In this paper, we discuss extensively the ray and wave dynamical properties in a spiral-shaped microcavity. The spiral-shaped microcavity has a special geometry in which the quasiscarred resonances dominate at some $n$ values [31]. The aim of this paper is to understand the quasiscarring phenomenon through the ray and wave dynamical properties. In Section 2, we introduce the steady probability distribution (SPD) and explain the ray dynamical properties based on the SPD in the spiral-shaped microcavity. We present resonance patterns and discuss about the quasiscarring phenomenon in Section 3, and in Section 4 the bouncing position of the quasiscarred patterns are explained through a semiclassical quantization condition. A brief comparison between the ray and the wave dynamical results is given in Section 5, and we conclude in the final section.

\section{Ray dynamics}

In this section, we first explain the steady probability distribution (SPD) which is a distribution in phase space $(s, p), s$ is the boundary coordinate and $p$ is its conjugate variable defined by $p=\sin \theta$, where $\theta$ is the incident angle (see Fig. 1 ). The SPD characterizes both the dynamical behaviors and the openness of the chaotic dielectric microcavities $[31,30,32]$. The basic concept of the SPD can be directly applicable to

other open systems with chaotic dynamics. We discuss the ray dynamical properties of the spiral-shaped microcavity based on the SPD, and show that the periodic orbits in 


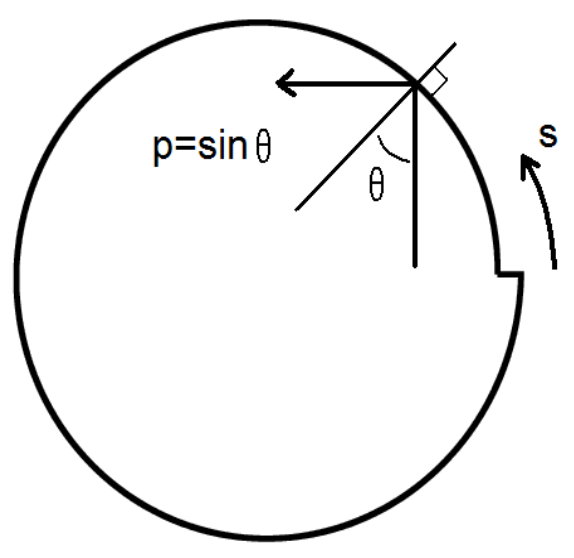

Figure 1. The spiral-shaped microcavity with $\epsilon=0.1$. It is fully chaotic and asymmetric. The phase space coordinates $(s, p)$ are shown.

the spiral-shaped cavity have at least one bounce from the notch.

\subsection{Steady probability distribution (SPD)}

The dielectric microcavity is an open system, which is an important difference from typical billiards, closed systems. At the boundary of the dielectric cavity the internal waves can partially transmit according to the Fresnel equations, thus the energy confined in the cavity would decrease with time. When the boundary geometry gives compeletely chaotic dynamics, due to this leaky property, the ray trajectory starting an arbitrary initial point $\left(s_{0}, p_{0}\right)$ cannot survive infinitely long time so that it cannot fills the whole phase space evenly as in the billiard case. Even in the case that $\left(s_{0}, p_{0}\right)$ locates in the total internal reflection region $\left(1 / n<\left|p_{0}\right|<1\right)$, the ray would diffuse along unstable manifolds and eventually reach the open region $\left(0<\left|p_{0}\right|<1 / n\right)$ and partially escapes. As a result, when we consider an ensemble of initial points, the rays distribute, after a transient time, on some structure of unstable manifolds containing the open property of the systems in the phase space, which is the basic concept of the SPD and is very useful to understand the resonance properties [31, 30].

The basic physical object characterizing the dynamical properties in an open system is the survival probability distribution $\tilde{P}(s, p, t)[32]$. Its integration over the phase space would give the survival probability $\tilde{P}(t)$ and $\tilde{P}(t)=\int d s d p \tilde{P}(s, p, t) \leq 1$ where the equality hold only at $t=0$ due to the energy loss by emission in the dielectric cavity. The initial distribution can be arbitrary when the system is fully chaotic, but here we consider a uniform initial distribution, $\tilde{P}(s, p, t=0)=2 / s_{m}$, where $s_{m}$ is the total length of the perimeter of the cavity. The confined energy and escape time distribution 


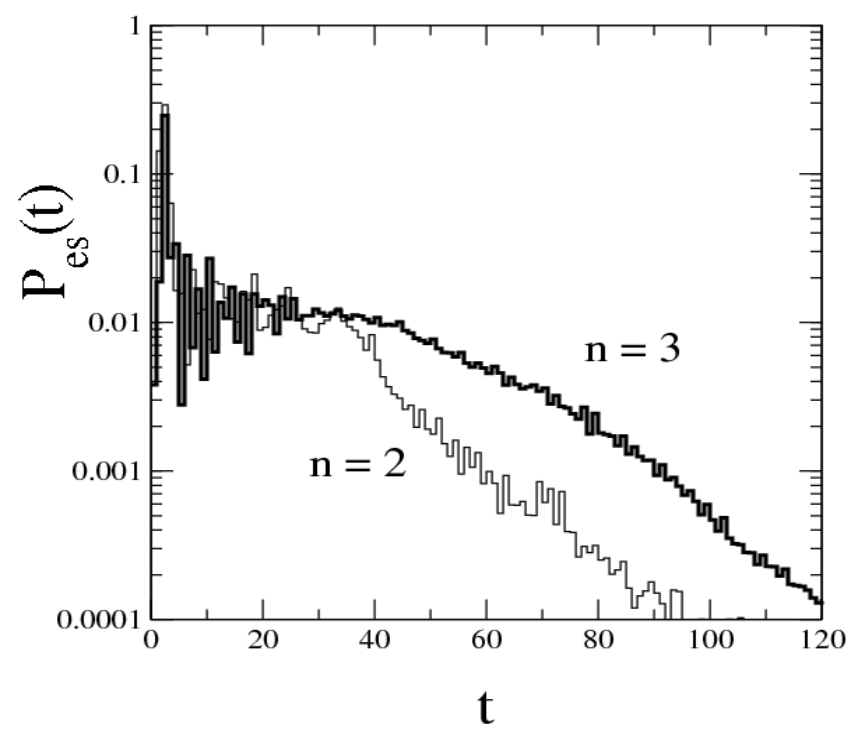

Figure 2. The escape time distributions $P_{e s}(t)$ when $n=2$ and 3 in the spiral-shaped microcavity with $\epsilon=0.1$. These show exponential long time behaviors, which is typical in fully chaotic systems.

can then be described by

$$
\begin{aligned}
& \mathcal{E}(t)=\mathcal{E}_{0} \int d s d p \tilde{P}(s, p, t), \\
& P_{e s}(t)=\frac{1}{\langle d\rangle} \int d s d p \tilde{P}(s, p, t) \mathcal{T}(p),
\end{aligned}
$$

where $\mathcal{E}_{0}$ is the initial energy confined in the cavity, and $\langle d\rangle$ is the average length of ray segment between two successive bounces. Here, the length of the trajectory can be regarded as time. $\mathcal{T}(p)$ is the transmission coefficient, which has nonzero value in the range of $-p_{c}<p<p_{c}\left(p_{c}=\sin \theta_{c}=1 / n\right)$, given by Fresnel's equation [33],

$$
\mathcal{T}=\frac{4 n \cos \left(\theta_{i}\right) \cos \left(\theta_{t}\right)}{\left(n \cos \left(\theta_{i}\right)+\cos \left(\theta_{t}\right)\right)^{2}},
$$

for TM wave where $\cos \left(\theta_{i}\right)=\sqrt{1-p^{2}}$ and $\cos \left(\theta_{t}\right)=\sqrt{1-n^{2} p^{2}}$ by Snell's law. Since the confined energy decreases by the ray transmission through cavity boundary, the relation between the confined energy and the escape time distribution is given by

$$
\frac{d \mathcal{E}(t)}{d t}=-\mathcal{E}_{0} P_{e s}(t)
$$

The above equations are generally satisfied by construction.

It is well known that in hyperbolic chaotic scattering the survival probability of a particle in the scattering region decays exponentially with time, while in nonhyperbolic chaotic scattering does algebraically due to the stickiness of KAM surfaces [34, 35, $36,37]$. The same argument holds in dielectric cavities. If the internal ray dynamics 


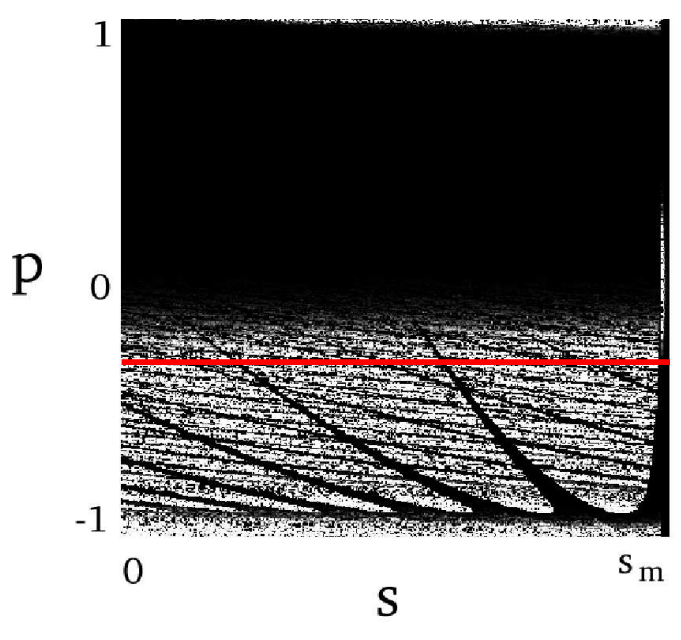

(a)

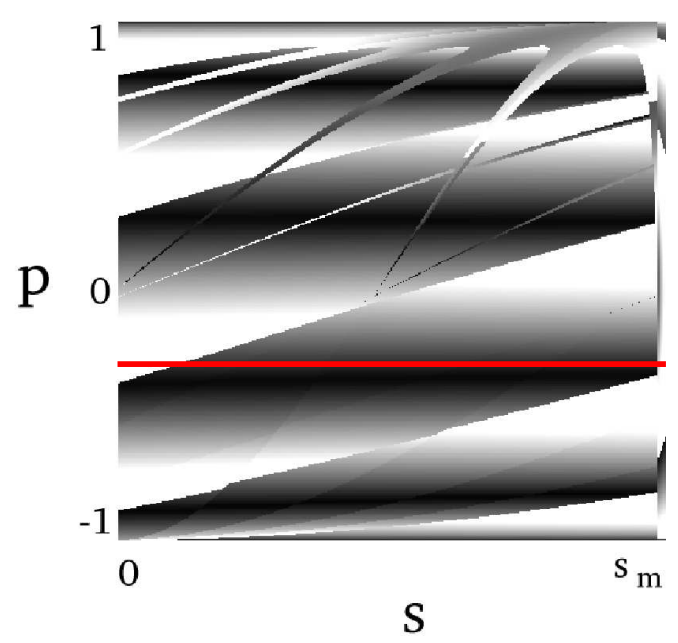

(b)

Figure 3. (a) The steady probability distributions $P_{s}(s, p)$, when $n=3$. (b) The distance distributions $d_{m=5}(s, p)$ after five bounces. The similar distribution for $n=2$ case is shown in Fig. 2 in [31]. From the comparison between $P_{s}(s, p)$ and $d_{m}(s, p)$, it is clear that the partially reflected rays near $p=-p_{c}$ (red line) make roughly (not exact) a triangle shape $(n=2)$ and a star shape $(n=3)$ trajectories.

is chaotic, then the survival probability and the escape time distribution $P_{e s}(t)$ have exponential tails [32]. The exponential tail of $P_{e s}(t)$ implies that the survival probability distribution $\tilde{P}(s, p, t)$ can separate into time part and phase space part, i.e.,

$$
\tilde{P}(s, p, t)=B(t) P_{s}(s, p) \text {. }
$$

Since the phase space part $P_{s}(s, p)$ is statistically invariant with time, it is called as the steady probability distribution.

Substituting Eq.(5) into Eqs.(1), (2) and, in turn, into Eq.(4), we can obtain the exponential time behavior, $B(t)=\exp (-\gamma t)$. The decay rate $\gamma$ is given by

$$
\gamma=\frac{1}{\langle d\rangle} \int d s d p P_{s}(s, p) \mathcal{T}(p) .
$$

We get, therefore, the exponential behaviors of the confined energy and the escape probability distribution at the long time tail,

$$
\begin{aligned}
& \mathcal{E}(t)=\mathcal{E}_{0} \exp (-\gamma t), \\
& P_{e s}(t)=\gamma \exp (-\gamma t) .
\end{aligned}
$$

We emphasize that in chaotic microcavity systems the steady probability distribution $P_{s}(s, p)$ plays a central role to characterize the long time behavior of the ray dynamics which is associated with the patterns of high $Q$ resonances [30]. The near field and far field distribution based on the ray dynamics are then expressed as

$$
\begin{aligned}
& P_{\text {near }}(s) \propto \int d p P_{s}(s, p) \mathcal{T}(p), \\
& P_{\text {far }}(\phi) \propto \int d s d p P_{s}(s, p) \mathcal{T}(p) \delta(\phi-f(s, p)),
\end{aligned}
$$


where the far field angle $\phi$ is given as $f(s, p)$ determined by the geometry of boundary and Snell's law. The refraction angle distribution is

$$
P_{r e f}\left(\theta_{t}\right) \propto \int d s d p P_{s}(s, p) \mathcal{T}(p) \delta\left(\theta_{t}-g(p)\right),
$$

where $g(p)=\arcsin (n p)$. Since the steady probability distribution $P_{s}(s, p)$ illustrates the long time behavior of the ray dynamics in the dielectric cavity, it is natural to expect that $P_{s}(s, p)$ would give some information about the pattern formation of resonances with relatively high $Q$ which are supported mainly by the rays having long trajectories before escaping. These relationship will be discussed in Section 5 .

\subsection{Spiral-shaped boundary geometry}

The spiral-shaped microcavity has been introduced by Chern et al.[4] to generate unidirectional lasing emission, and the boundary shape is given by

$$
r(\phi)=R\left(1+\frac{\epsilon}{2 \pi} \phi\right) .
$$

in polar coordinates $(r, \phi)$, where $R$ is the radius of the spiral at $\phi=0$ and $\epsilon$ is the deformation parameter and we set $\epsilon=0.1$ throughout the paper (see Fig. 1). The spiral geometry of the cavity has interesting aspects compared with typical shapes treated in the previous studies. First the internal ray dynamics is fully chaotic. A trajectory starting from an arbitrary initial point $\left(s_{0}, p_{0}\right)$ fills evenly the whole phase space $(s, p)$ when refractive escapes are ignored. Second, it is totally asymmetric, i.e., does not have any continuous or discrete symmetries, which results in the chirality of the SPD and even resonance patterns (see Fig. 6 and 7).

The internal rays in the spiral-shaped cavity have a simple flow. Consider a ray circulating clockwisely $\left(p_{0}<0\right)$. As long as the ray circulates clockwise, there is no chance to bounce off from the notch and the negative incident angle increases gradually and, after some bounces, change its circulating direction, i.e., circulates counterclockwise $(p>0)$. Then the incident angle of the ray increases ( $p$ increases) gradually until it bounces off from the notch. After that, the ray again circulates clockwise and repeats the above description. Note that the $p$ value of the ray always increases except the case that the ray hit the notch.

In order to obtain numerical results for $P_{e s}(t)$ and $P_{s}(s, p)$, let us consider an ensemble of initial points which are uniformly distributed on the $700 \times 700$ grid points over the whole phase space. The rays starting from the initial points would suffer bounces from the boundary, and some rays with $|p|>1 / n$ are totally reflected and the other rays are partially transmitted through the boundary with probability $\mathcal{T}(p)$. After this process the points are rearranged and weighted. If the transmission probabilities of each rays are summed up in the time interval $(t, t+\delta)$, then we can get numerically the escape time distribution $P_{e s}(t)$. The numerical results are shown in Fig. 2 for $n=2$ and 3 cases. A characteristic feature of $P_{e s}(t)$ is the exponential decay behavior, which is typical in fully chaotic systems as mentioned before. 
Figure 3(a) shows the approximate steady probability distribution $P_{s}(s, p)$ for $n=3$ (the similar distribution for $n=2$ is shown in Fig. 2(a) in [31]), given by normalizing the survival probability distribution $\tilde{P}(s, p, t)$ in the time range of $57<t<60$ for the uniform ensemble of initial points. The structure of the approximate $P_{s}(s, p)$ is almost invariant in other time ranges of the exponential region in Fig. 2. The restricted distribution of the SPD in $p<0$ region means that most of the rays, after the transient time, circulate clockwisely, which is very different from the stadium shape cavity [32], and means the strong chirality of the spiral-shaped microcavity. This chiral property will be also appearred in resonance patterns in the next section. In the details of the SPD we can see tentacular structure and some fine structures. These structures reveal the result of mixing of two structures, one is of the unstable manifolds given by the boundary geometry and the other is of the openness of dielectric cavity determined by Fresnel equations. From the SPDs it is clear that the rays escape refractively at the notch and just above the critical line $\left(-p_{c}=-1 / n\right)$.

Since the rays near the critical line $p=-p_{c}$ play a central role in the formation of the quasiscarred resonances [31], it is necessary to follow the ray trajectories and see what type of polygon is similar to those. In fact, this is easily expected from a consideration of circular cavity under an assumption that the deformation parameter $\epsilon=0.1$ would not give a big change from the case. For $n=2$ case, the critical angle $\theta_{c}$ is $\arcsin (1 / 2)=\pi / 6$ and therefore the ray with the incident angle $\theta_{c}$ follows the triangular periodic orbit in a circular cavity. Also, for $n=3$ case, $\theta_{c}=\arcsin (1 / 3) \simeq 0.3398$ is similar to $\pi / 10 \simeq 0.314$, the incident angle required to make a star-shaped periodic orbit in the circular cavity. To check this expectation, we show the distance distribution, defined as $d_{m}\left(s_{i}, p_{i}\right)=\left(s_{f}-s_{i}\right)^{2}+\left(p_{f}-p_{i}\right)^{2}$ where $\left(s_{i}, p_{i}\right)$ is the initial position and $\left(s_{f}, p_{f}\right)$ being the position after $m$ bounces. We note that in Fig. $3(\mathrm{~b})$ the critical line $p=-p_{c}$ lies on the dark region representing the initial positions which give low values of $d_{5}$ ( $d_{3}$ is shown in Fig. 2 (b) in [31]). Therefore, we confirm that the rays reflected near the critical line $p=-p_{c}$ are would make roughly triangle shape $(n=2)$ and star shape $(n=3)$ geometries. As discussed later in Section 3 , the imprint of this fact appears apparently in resonance patterns.

\subsection{Periodic orbits in the spiral-shaped geometry}

Here, we prove the absence of periodic orbits of simple geometry such as triangle and star shapes. In other words, we have to prove nonexistence of periodic orbits without bouncing the notch, i.e., all periodic orbits must bounce the notch more than once. Let us define $D_{i}$ as the distance between the origin and the $i$ th ray segment determined by a line from $s_{i-1}$ to $s_{i}$ on the boundary (See Fig. 4(a)). Now, consider two different cases. The first case is the arbitrary $i$ th ray segment move around the origin in counterclockwise sense and the other is clockwise case.

Case-1: If the $i$ th ray-segment move counterclockwisely around the origin, the distance $D_{i}$ is always increasing after bouncing off from the boundary. It is easy to 


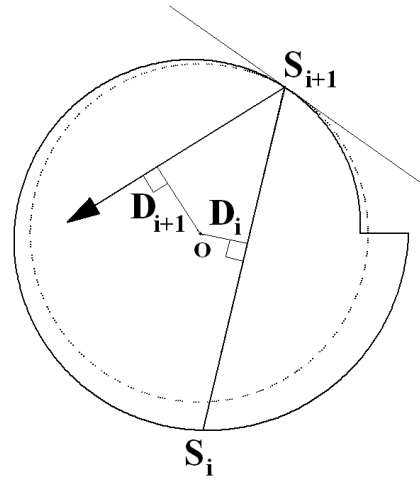

(a)

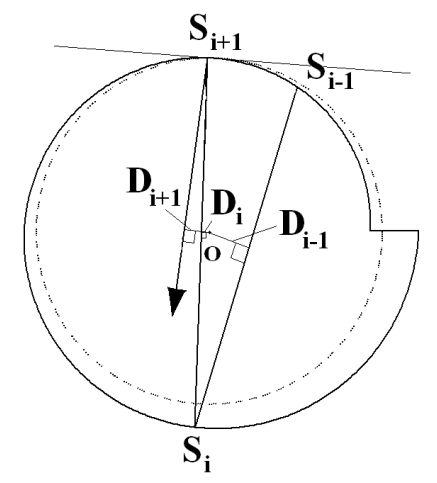

(b)

Figure 4. (a) The distance $D_{i}$ always increases for counterclockwisely propagating rays. (b) The clockwisely propagating rays eventually change its direction of rotation as far as it does not hit the notch part. (The dotted line is a virtual circle passing $i$ th bouncing point and having common origin with the spiral)

see by introducing virtual circle passing the $i$ th bouncing point and having common origin with the spiral as is shown in Fig. 4(a). This condition is satisfied by any points on the spiral perimeter since $r(\phi)$ is always increasing as $\phi$ increases. So, we get the following relation; $D_{i}<D_{i+1}<D_{i+2} \cdots$. If there is a periodic orbit of $N$-bouncing on the spiral perimeter, it must satisfy the relation; $D_{i}=D_{i+N}$. This relation definitely contradictory with the previous one. So, if the ray-segment move counterclockwisely around the origin, it never make a periodic orbit without bouncing at the notch.

Case-2: If the $i$ th ray-segment move clockwisely around the origin, we can say that the distance $D_{i}$ is always decreasing after bouncing while the ray-segment move clockwisely around the origin. However, unlike the Case- 1 , when $D_{i}$ becomes close to zero, the ray-segment turns its rotational direction from clockwise to counterclockwise as shown in Fig. 4.(b). Then, the Case-2 reduces to the Case-1. Such a turning always occurs in clockwise case eventually as far as it does not hit the notch. As a result, we can conclude there is no periodic orbit without bouncing the notch.

\section{Wave dynamics}

In this section, we present resonance positions around $\operatorname{Re}(n k R) \simeq 110$, and corresponding resonance patterns in the spiral-shaped microcavity for $n=2$ and 3 . This direct numerical calculation of the resonances can improve the physical insight into the pattern formation in microcavities through the comparison with the ray dynamical analysis in the previous section.

We use the boundary element method [38] to get the resonance positions and patterns. Since the spiral shape has two nonanalytic points which invoke the infinite curvature problem in the boundary element method, we round the corners with circle 


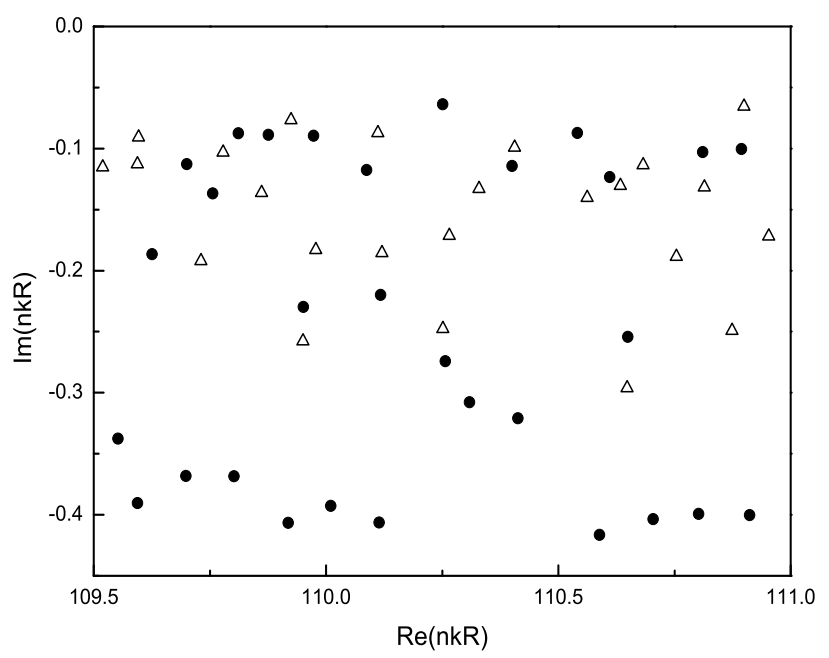

Figure 5. The resonance positions. The solid circles and the open triangles represent resonance positions for $n=2$ and 3 , respectively.

with a radius $r$. The radius $r$ should be much less than $\lambda=2 \pi / \operatorname{Re}(n k R)$ so that the wave cannot be aware of the rounding. In principle, it is possible to get all resonances in a finite range of $\operatorname{Re}(n k R)$, but it requires much time in practical calculations. Therefore, we take the following strategy to find a part of resonances. We consider a tension function $T(n k R)$ [39] which is a measure indicating how far away $k R$ is from the resonance positions, so if $T\left(n k_{i} R\right)=0$ then the $n k_{i} R$ is just a resonance position. We first evaluate the tension on the three straight lines in the complex $k R$ plane which are $\operatorname{Im}(k R)=0.04,0.08$, and 0.12 in the range of $109.5<\operatorname{Re}(n k R)<111$. Then, starting from the positions giving local minimums of the tension on the lines, we apply Newton's method to find the resonance positions. In our calculations, we divide the boundary into 1000 elements, i.e., 900 elements on the perimeter of the spiral part and 100 elements on the notch part, which approximately correspond to more than 8 elements per wavelength $\lambda$ on the boundary.

\subsection{Resonance positions}

Figure 5 shows the resonance positions for $n=2$ and 3 in the spiral-shaped microcavity. We find 30 resonances for $n=2$ and 24 resonances for $n=3$. These numbers are surely much smaller than the expected total numbers, i.e., about 91 , which is estimated by the modified Weyl's theorem [40,41]. These numbers of resonances, however, are enough to reveal resonance properties of the spiral-shaped microcavity.

A notable feature shown in Fig. 5 is that the resonances for $n=3$ (the open triangles) distribute in the upper part of the complex plane in comparison with those for $n=2$ (the solid circles). This implies that internal waves can be more effectively 


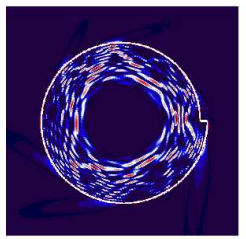

(a)

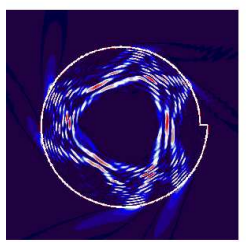

(f)

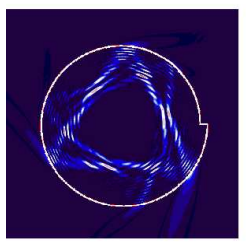

(k)

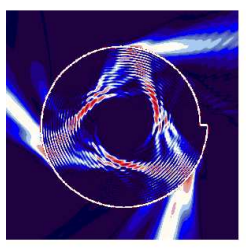

(p)

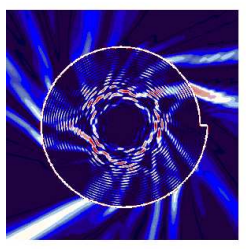

(u)

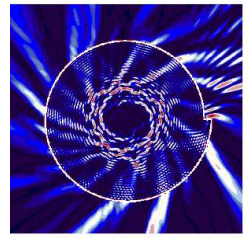

(z)

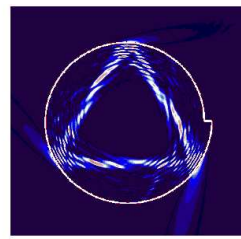

(b)

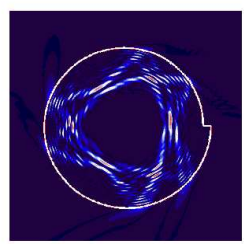

(g)

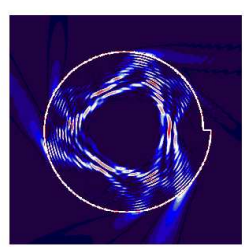

(I)

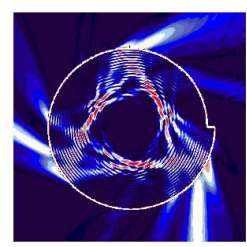

(q)

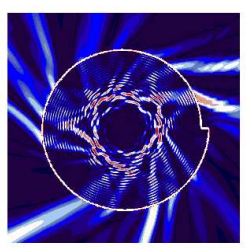

(v)

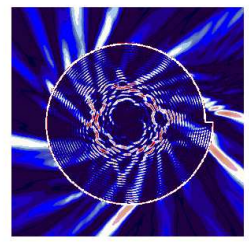

(aa)

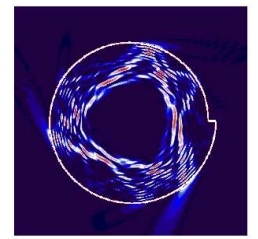

(c)

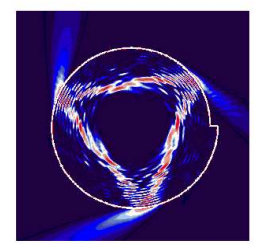

(h)

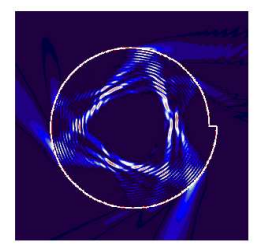

(m)

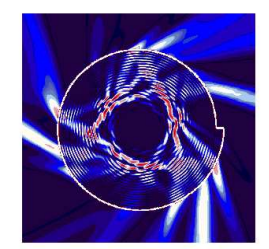

(r)

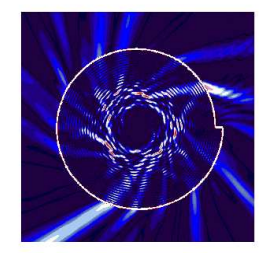

(w)

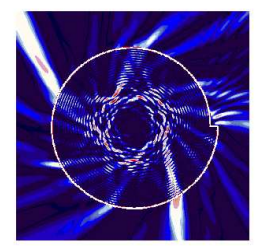

(bb)

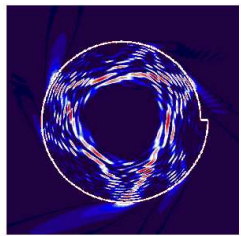

(d)

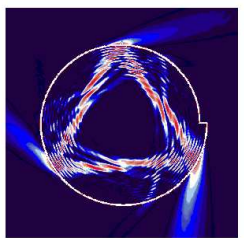

(i)

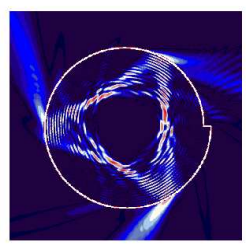

(n)

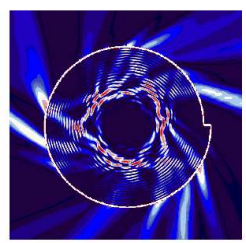

(s)

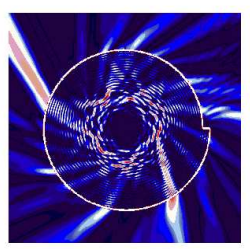

(x)

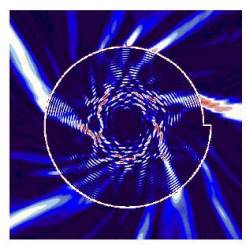

(cc)

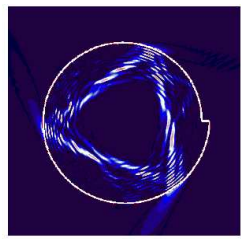

(e)

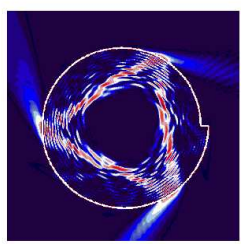

(j)

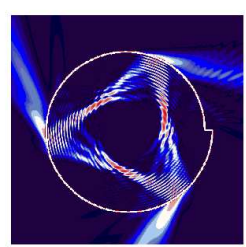

(o)

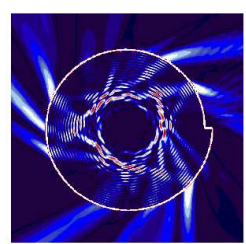

(t)

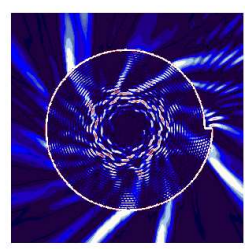

(y)

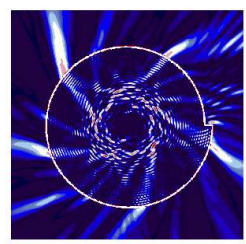

(dd)

Figure 6. (Color online) Resonance patterns for $n=2$ case. These correspond to the solid circles in Fig. 5, and are arranged in the increasing order of $|\operatorname{Im}(n k R)|$. (The intensity of the fields is normalized by scaling the maximum intensity inside the cavity as one.) The calculated resonance positions are (a) $n k R=(110.25,-0.0637)$, (b) $(110.54,-0.0871)$, (c) $(109.81,-0.0873)$, (d) $(109.88,-0.0887)$, (e) $(109.97,-0.0896)$, (f) $(110.89,-0.1003),(g)(110.81,-0.1029)$, (h) $(109.70,-0.1128)$, (i) $(110.40,-0.1142),(j)$ (110.09,-0.1174), (k) (110.61,-0.1233), (l) (109.76,-0.1367), (m) (109.63,-0.1866), (n) $(110.12,-0.2198)$, (o) $(109.95,-0.2297),(p)(110.65,-0.2543),(q)(110.26,-0.2740),(\mathrm{r})$ $(110.31,-0.3078),(\mathrm{s})(110.41,-0.3208),(\mathrm{t})(109.55,-0.3375),(\mathrm{u})(109.70,-0.3680),(\mathrm{v})$ $(109.80,-0.3684),(\mathrm{w})(109.59,-0.3904),(\mathrm{x})(110.01,-0.3927),(\mathrm{y})(110.80,-0.3992),(\mathrm{z})$ (110.91,-0.4002), (aa) (110.70,-0.4036), (bb) (110.11,-0.4063), (cc) (109.92,-0.4066), (dd) $(110.59,-0.4164)$, respectively. 
confined by the microcavity when the reflective index $n$ is increased. The open region is reduced with increasing $n$, since the open region is $-1 / n<p<1 / n$, and the transmission coefficient $\mathcal{T}(p)$ in Eq. (3) decreases, e.g., for the normal incident case, $\mathcal{T}(p=0)=4 n /(n+1)^{2}$. Therefore, as $n$ increases, the resonance positions would distribute closer to the real axis.

\subsection{Resonance patterns: quasiscarred resonances}

The resonance patterns in the case of $n=2$ and 3 corresponding to the resonance positions in Fig. 5 are shown in Fig. 6 and Fig. 7, respectively. In the resonance patterns we note that the basic localized structures of the resonances with relatively high $\mathrm{Q}$ are triangular and star shapes for $n=2$ and 3, respectively, which is closely related to the fact that the rough polygons drawn by the rays near the critical line $p=-p_{c}$ are triangle and star as discussed in the previous section. These basic structures appear in about half of the resonances. Since the localized patterns of resonances are not supported by any exact unstable periodic orbit, we call these quasiscarred resonances.

The figures in Fig. 6 and Fig. 7 are arranged in the order of the $|\operatorname{Im}(n k R)|$ value, i.e., the upper is the smaller $|\operatorname{Im}(n k R)|$. From these patterns we can find a general rule about the relationship between the intensity distributions of patterns and $|\operatorname{Im}(n k R)|$ values or $Q$ values. The high $Q$ resonances with smaller $|\operatorname{Im}(n k R)|$ show WGM-type patterns, resulting larger hollow region at the central part. As the $|\operatorname{Im}(n k R)|$ increases, resonances start to show clear localization on triangle or star shape geometry, i.e., quasiscarred patterns. As the $|\operatorname{Im}(n k R)|$ further increases, quite leaky patterns start to appear that show many beams emitted from the cavities and result smaller hollow region at the central part. This implies that the leaky resonances are supported by the part far above the critical line $p=-p_{c}$ (smaller incident angle), while the quasi-scarred resonances are done with the part near the line $p=-p_{c}$ in the phase space. The quasiscarred resonances clearly show the chirality, i.e., the internal waves circulate only clockwise (see the Husimi functions in Fig. 8), which is consistent with the SPD structures in Fig. 3(a).

Now, let us consider the quasiscarring phenomenon in the dielectric microcavity. From the results of the ray dynamical analysis and the resonance patterns, one can simply expect that, although there is no exact unstable periodic orbit matched to the quasiscarred patterns, slightly diffractive trajectories can make a closed orbit, and the quasiscarring phenomenon would be based on the waves propagating along the diffractive closed orbit and satisfying constructive interference condition. This might be the case in the closed billiard case, but in the dielectric microcavity case only considering the diffractive waves is not enough to explain the numerical results. First, wave diffraction can occur equivalently in a closed spiral-shaped billiard system, but it is not easy to find quasiscarred eigenfunctions in the closed cavity $[42,43]$. Note that about half of the resonances in Fig. 6 and 7 are quasiscarred in the open dielectric microcavity. Second, the dominant pattern of quasiscarring is triangles for $n=2$ and stars for $n=3$. With 


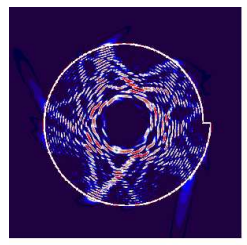

(a)

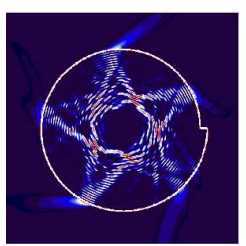

(f)

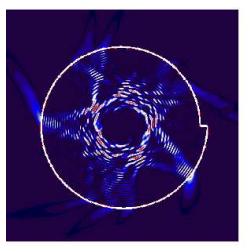

(k)

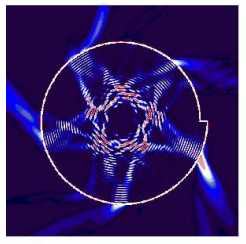

(p)

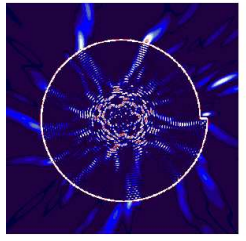

(u)

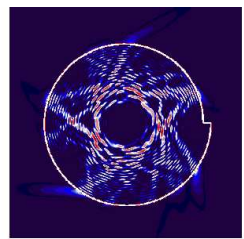

(b)

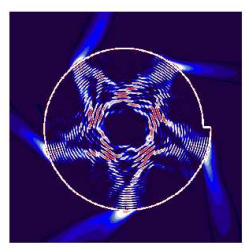

(g)

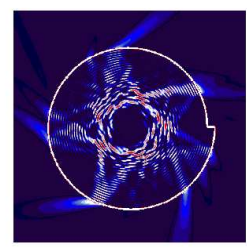

(I)

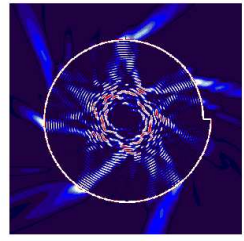

(q)

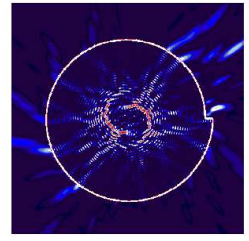

(v)

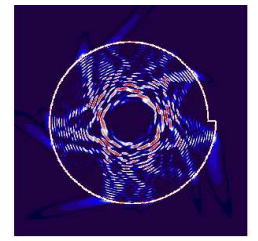

(c)

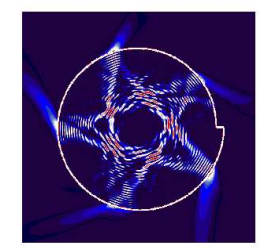

(h)

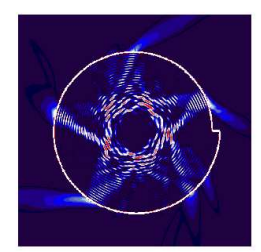

(m)

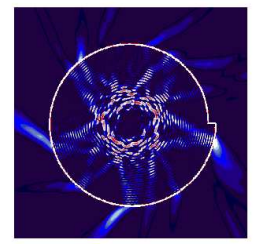

(r)

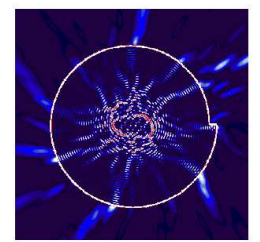

(w)

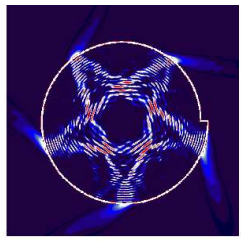

(d)

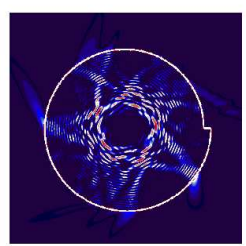

(i)

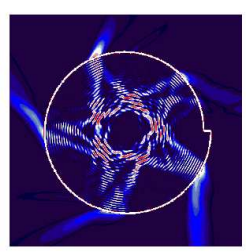

(n)

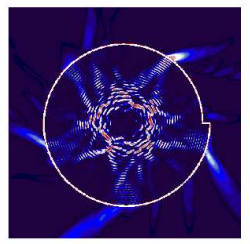

(s)

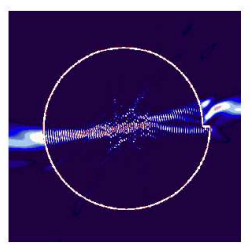

(x)

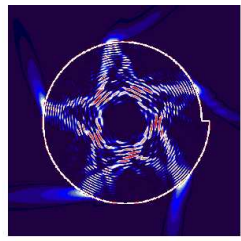

(e)

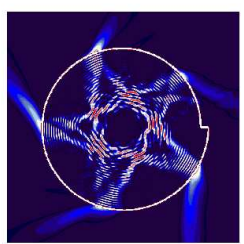

(j)

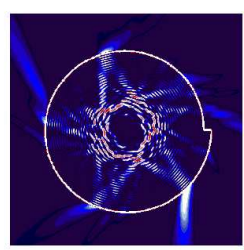

(o)

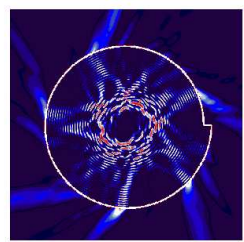

(t)

Figure 7. (Color online) Resonance patterns for $n=3$ case. These correspond to the open triangles in Fig. 5, and are arranged in the increasing order of $|\operatorname{Im}(n k R)|$. (The intensity of the fields is normalized by scaling the maximum intensity inside the cavity as one.) The calculated resonance positions are (a) $n k R=(110.90,-0.0654)$, (b) $(109.92,-0.0766)$, (c) $(110.11,-0.0873)$, (d) $(109.60,-0.0907)$, (e) $(110.41,-0.0991)$, (f) $(109.78,-0.1030),(g)(109.59,-0.1127),(h)(110.68,-0.1135)$, (i) $(109.52,-0.1152),(j)$ (110.63,-0.1303), (k) (110.81,-0.1318), (l) (110.33,-0.1330), (m) $(109.86,-0.1361),(\mathrm{n})$ $(110.56,-0.1403)$, (o) $(110.27,-0.1712)$, (p) $(110.95,-0.1718)$, (q) $(109.98,-0.1829)$, (r) $(110.12,-0.1855),(\mathrm{s})(110.75,-0.1885)$, (t) $(109.73,-0.1920)$, (u) $(110.25,-0.2477),(\mathrm{v})$ $(110.87,-0.2491),(\mathrm{w})(109.95,-0.2578),(\mathrm{x})(110.65,-0.2962)$, respectively. 

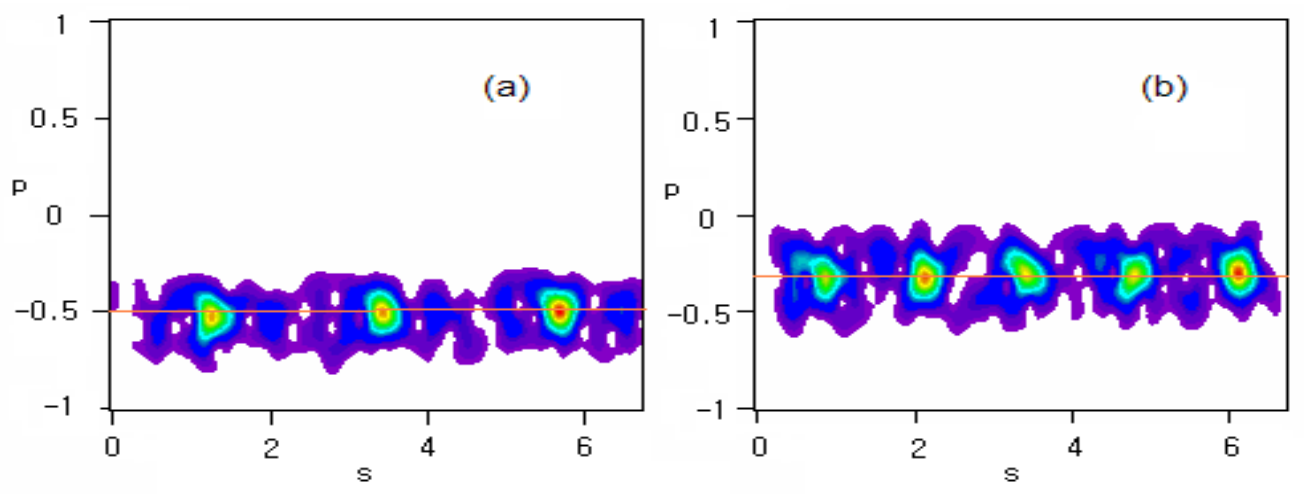

Figure 8. (Color online) (a) The incident Husimi function of the quasiscarred resonance (j) in Fig. 6. (b) The incident Husimi funcution of the quasiscarred resonance (j) in Fig. 7. The solid line denotes the critical line, $p=-p_{c}=-1 / n$.

only diffractive waves, one cannot explain why the quasiscarring pattern changes with the refractive index $n$.

Therefore, in order to understand quasiscarring in dielectric microcavities, we should take account of the unique properties of the dielectric openness. It is emphasized that the localization of quasiscarred resonances appears near the critical line $p=-p_{c}$ as shown clearly in the Husimi functions [44] in Fig. 8. This implies that the waves with an incident angle near $\theta_{c}$ are effective to form the quasiscarred resonance. In fact, recent studies $[30,45]$ pointed out that the wave beam propagation at a dielectric interface shows very different behavior from the expected ray trajectory, and the difference reaches a maximum when the incident angle of the wave beam crosses the critical angle $\theta_{c}$. The difference is described by the Goos-Hänchen shift $[46,47]$ and the Fresnel filtering effect [48]. The Goos-Hänchen shift is the distance between the incident beam center and the reflected beam center on the dielectric interface, and the Fresnel filtering effect gives the result that the reflection angle of the beam is slightly greater than the incident angle of the beam due to the partial transmission. In the triangular patterns in Fig. 6, when connecting the bouncing positions, the reflection angle is greater than the incident angle by about $1.7^{\circ}$. It is now believed that the unique properties in dielectric cavities play a central role in quasiscarring phenomenon shown in the spiral-shaped microcavity.

\section{Bouncing positions in quasiscarred resonances}

Now, we consider bouncing positions of the triangle formed in quasi-scarred resonances when $n=2$ in the spiral-shaped dielectric microcavity. These have a definite dependence on their $\operatorname{Re}(n k R)$ values (see Fig. 12). We assume that the triangle in a quasi-scarred 


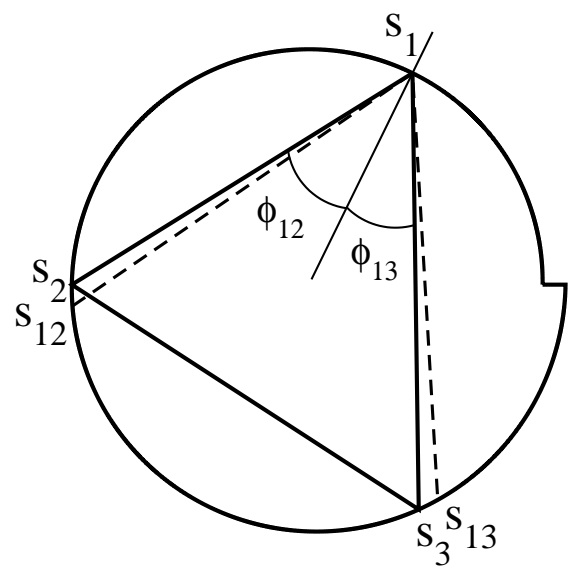

Figure 9. Schematic diagram for quantifying the deviation from the periodic orbit condition. The trajectory satisfying reflection law, with an incident angle $\left(\phi_{12}+\phi_{13}\right) / 2$, is denoted by dashed lines.

resonance would have minimum deviation from the periodic orbit condition governed by the reflection law, and satisfy the semiclassical quantization rule including the effect of Maslov index.

\subsection{Triangles showing minimum deviation from periodic orbit condition}

We quantify the deviation from the periodic orbit condition as $\alpha$. Let $s_{i}(i=1,2,3)$ be the bouncing positions of a triangle, from the angles $\left(\phi_{i j}, \phi_{i k}\right)$ to the normal line on the boundary at $s_{i}$, we can define $p_{i j}=\sin \left(\phi_{i j}\right), p_{i k}=\sin \left(\phi_{i k}\right)$, and $p_{i}=\sin \left(\frac{\phi_{i j}+\phi_{i k}}{2}\right)$ (here $i, j, k$ are cyclic). Also we get the new positions $s_{i j}, s_{i k}$ as the next positions of $\left(s_{i}, p_{i}\right)$ and $\left(s_{i},-p_{i}\right)$, respectively (see Fig. 9 ). Then we define partial deviations of the triangle given by $\left(s_{1}, s_{2}, s_{3}\right)$ as

$$
\alpha_{i}=\left[\left(p_{i}-p_{i j}\right)\left(s_{j}-s_{i j}\right)\right]^{2}+\left[\left(p_{i}-p_{i k}\right)\left(s_{k}-s_{i k}\right)\right]^{2} .
$$

Total deviation, therefore, is the sum of these terms, $\alpha=\sum_{i=1}^{3} \alpha_{i}$. By definition, $\alpha$ becomes zero when the triangle is a periodic orbit.

From a numerical calculation, we obtain the triangles which have local minimum values of $\alpha$. The local minimum value linearly decreases as $s_{1}$ increases, and the corresponding partial deviations are shown in Fig. 10. We can see the inequality, $\alpha_{1}<\alpha_{2}<\alpha_{3}$, and the $\alpha^{*}$ is introduced for convenience and defined as $\alpha^{*}=$ $\Delta p^{2}(\sqrt{3} R \arcsin (\Delta p))^{2}$ with $\Delta p=0.03$ and $R=1$, noting $p_{c}-\Delta p<p_{i j, i k}<p_{c}+\Delta p$. Figure 11 show the bouncing positions of the triangle showing the minimum deviation as a function of $s_{1}$. They are well fitted as a linear function of $s_{1}$,

$$
\begin{aligned}
& s_{2}\left(s_{1}\right) \simeq 1.0326 s_{1}+2.1104, \\
& s_{3}\left(s_{1}\right) \simeq 1.0656 s_{1}+4.3285 .
\end{aligned}
$$




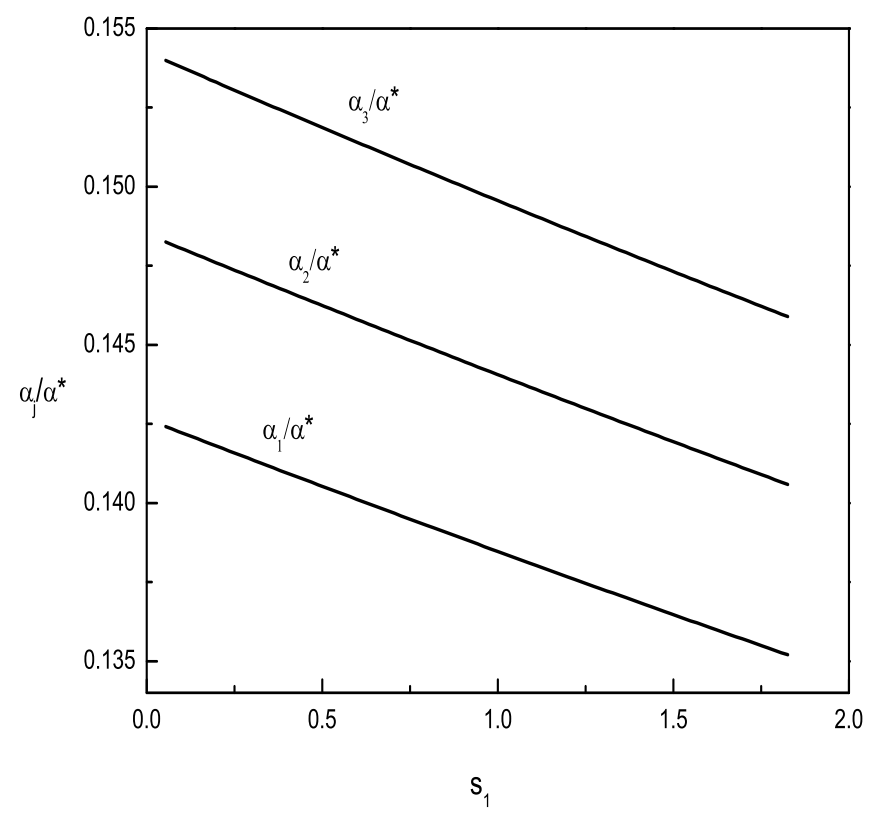

Figure 10. The minimum $\alpha\left(=\alpha_{1}+\alpha_{2}+\alpha_{3}\right)$ from the periodic obit condition (see Eq. (13)).

The corner angles of the triangles are almost invariant with $s_{1}$. The details are $p_{1} \simeq 0.516, p_{13} \simeq 0.529, p_{12} \simeq 0.503, p_{2} \simeq 0.5, p_{21} \simeq 0.513, p_{23} \simeq 0.487, p_{3} \simeq 0.484$, $p_{32} \simeq 0.497$, and $p_{31} \simeq 0.470$. The lengths of the line segments of the triangles increase with $s_{1}$ and if $l_{j}$ is defined by the line length between $s_{j}$ and $s_{i}$, then the triangles show $l_{2}<l_{1}<l_{3}$ as shown in Fig. 11 (b). The perimeter of the triangle, $L=l_{1}+l_{2}+l_{3}$, is well described by a linear function of $s_{1}$,

$$
L\left(s_{1}\right) \simeq 0.0815 s_{1}+5.3698 .
$$

These information of the triangles with minimum deviation from the periodic orbit will be used in the next subsection and enable us to express the result in an analytic form.

\subsection{Semiclassical quantization condition}

To be a triangular quasiscarred resonance obtained in the previous subsection, the waves should be exactly in phase after a round trip so that a constructive interference occurs and then the wave inside the cavity survives longer time. It is known that the total accumulated phase consists of three contributions: a dynamical phase, a pure quantum phase introduced by boundary conditions and a topological phase associated with the rotation of the manifolds [49]. In the present case, the dynamical phase is $n k L$ corresponding the phase developed during wave propagation, where $L$ is the perimeter 

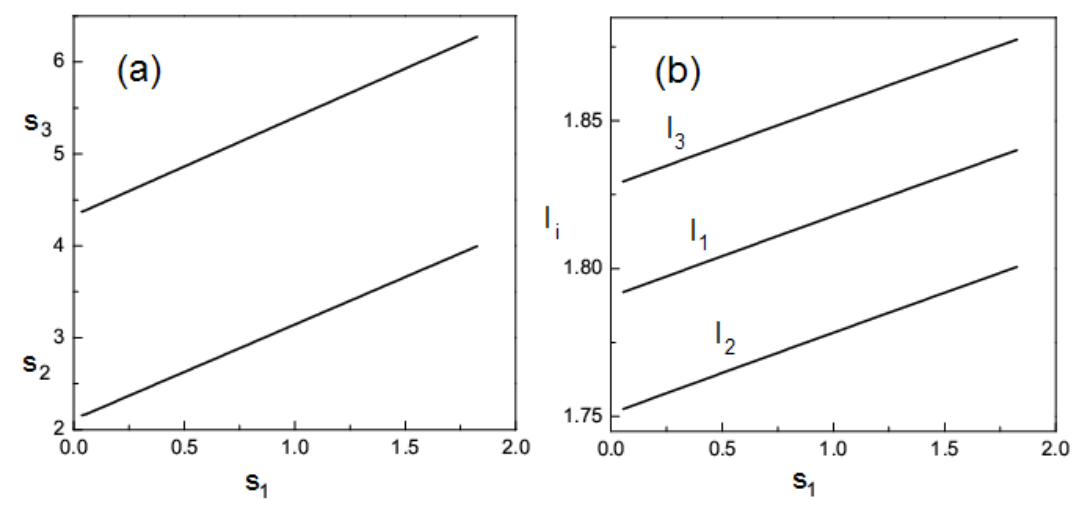

Figure 11. (a) The bouncing positions $\left(s_{1}, s_{2}, s_{3}\right)$ and (b) the lengths of line segments $\left(l_{1}, l_{2}, l_{3}\right)$ of the triangles giving the minimum $\alpha$.

of the triangle. The pure quantum phase arises when the wave is reflected from the boundary, but it is always accompanied with the topological phase. We note that there is a phase loss of $\pi$ due to Dirichlet boundary conditions while the phase does not change for Neumann conditions. In the dielectric cavity, the phase loss at the boundary varies from zero to $\pi$ as the incident angle goes from $\theta_{c}$ to $\pi / 2$ [33], i.e.,

$$
\phi(\theta)=\frac{2 \arctan \left(\sqrt{\sin ^{2} \theta-1 / n^{2}}\right)}{\cos \theta} .
$$

In the triangles with minimum deviation, the incident angles correspond to $\arcsin \left(p_{12}\right)$, $\arcsin \left(p_{23}\right)$ and $\arcsin \left(p_{31}\right)$. Only $\arcsin \left(p_{12}\right)$ is greater than $\theta_{c}$ and contributes to the phase loss. This phase loss varies slightly with $s_{1}$ as

$$
\phi\left(s_{1}\right) \simeq-0.000286 \pi s_{1}+0.0387 \pi .
$$

As mentioned before, this phase loss includes the topological phase, i.e., an increment of Maslov index by one when a reflection from the cavity occurs. Thus, the net phase loss by the pure quanutum phase would be $\phi-N_{b} \pi / 2$, where $N_{b}$ is the number of bounces, $N_{b}=3$ for the triangle. Finally, the topological phase is expressed by $\mu \pi / 2$ where $\mu$ is Maslov index, in the present case $\mu=N_{b}+\nu, \nu$ is the number of bounces from circular boundary, i.e., $\nu=3$ in our case. Therefore, the semiclassical quantization condition for the quasiscarred resonance is

$$
n k L\left(s_{1}\right)-\phi\left(s_{1}\right)-\nu \pi / 2=2 \pi m,
$$

where $m$ is an integer. Using Eqs.(15) and (17), we can express $s_{1}$ as a function of $k$ as

$$
s_{1}=\frac{2 \pi m-5.3698 n k+0.0387 \pi+3 \pi / 2}{0.0815 n k+0.000286 \pi} .
$$




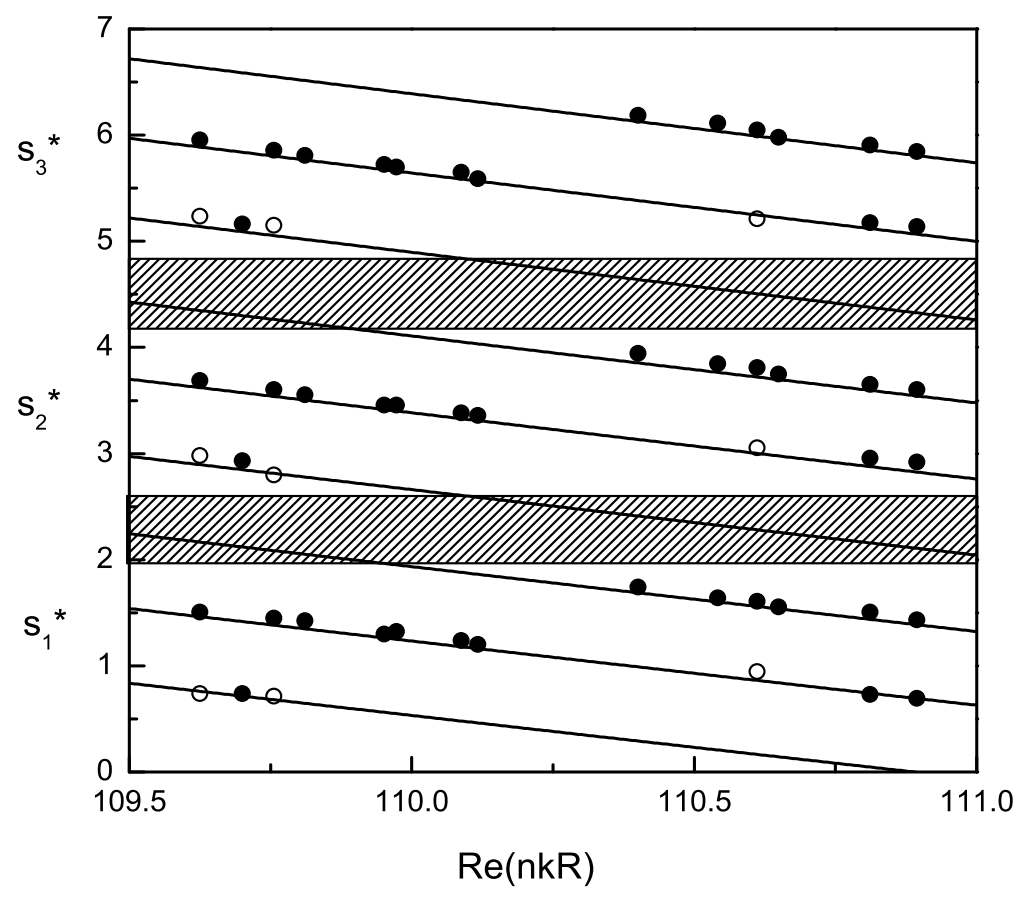

Figure 12. Variation of the bouncing positions of the triangular quasiscarred resonances of $n=2$ case in Fig. 6. The solid lines denote the results given by the semiclassical quantization condition. Three solid circles with the same $\operatorname{Re}(n k R)$ correspond to the main triangular pattern, and three open circles to the secondary triangular pattern in a quasiscarred resonance. The shaded regions indicate the forbidden zones which is consistent with the SPD structure.

So, for a given $n k$ value, we can obtain $s_{1}$ from the above equation, and then use Eq.(14) to get $s_{2}$ and $s_{3}$. The result is shown as solid lines in Fig. 12 where $m=94,95$, and 96 are used. The data points correspond to the center positions of intensity peaks in the boundary functions of the quasiscarred resonances shown in Fig. 6. The open circles represent the bouncing positions of the secondary triangle of the quasiscarred resonances showing two triangles. It is impressive that the semiclassical quantization condition gives very good agreement with the data points without any fitting parameter. Absence of bouncing positions near $s=2.2$ and 4.5 is consistent with the dark tentacular structure of the approximate steady probability distribution $P_{s}(s, p)$ in Fig. 2(a) of [31].

\section{Comparison between ray and wave dynamics}

As mentioned in section II, the far field and the near field distribution can be calculated ray dynamically from the SPD. The numerical results are shown as histograms in Fig. 13 for both $n=2$ and 3. Far field distributions $P(\phi)$ can also be obtained by taking an 

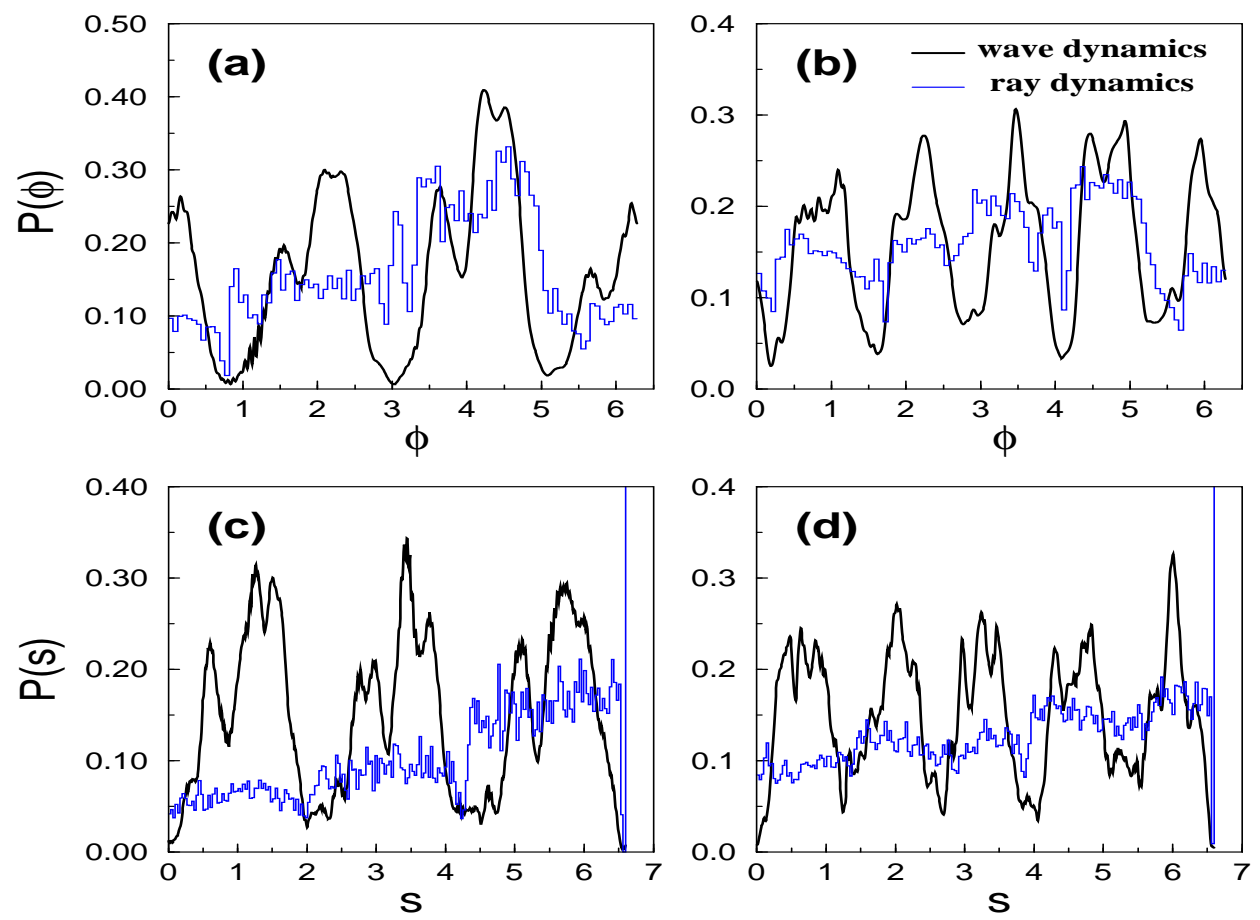

Figure 13. The far field distributions $P(\phi)$ and the near field distribution $P(s)$ are compared with the results from the ray dynamical analysis. (a) $P(\phi)$ for $n=2$, (b) $P(\phi)$ for $n=3$, (c) $P(s)$ for $n=2$, and (d) $P(s)$ for $n=3$. The ray dynamical results based on the SPD are blue histograms, and the wave dynamical results obtained from the resonance patterns are black solid lines.

average over the individual far field distribution of the resonances shown in Figs. 6 and 7. The individual far field distribution for a specific resonance is obtained by calculating intensities over a large circle (the radius used is 50R) and normalizing the intensities. For the near field distributions, the boundary wave functions obtained from the boundary element method calculations are used for the average process. In the average process, we exclude very leaky resonance modes because the SPD characterizes long time ray dynamical behaviors and corresponds to relatively high $\mathrm{Q}$ resonances. We have compared these results based on the resonance patterns (the solid lines in Fig. 13) with the results from the ray dynamical analysis (histograms in Fig. 13). We can see good qualitative agreements between them in spite of only limited number of resonances being used for calculations.

\section{Conclusion}

We study both the ray and wave dynamical properties in the dielectric spiral-shaped microcavity when $n=2$ and 3 . The ray dynamical property is well characterized by the steady probability distribution which contains the mixed characters of the ray dynamics and the openness of the dielectric cavities. The quasiscarred patterns in resonances 
are shown as triangle for $n=2$ case and star for $n=3$ case. These quasiscarring phenomenon can be understood by taking account of the unique property of wave propagation at the dielectric boundary. We also explain the bouncing positions of the quasiscarred pattern using the semiclassical quantization condition including the effect of Maslov index, and show a qualitative agreement between the ray and wave dynamical results in both far field and near field distributions.

\section{Acknowledgments}

This work is supported by the Creative Research Initiatives (Quantum Chaos Applications) of MOST/KOSEF. C. M. Kim is partially supported by Sogang Research Grant of 20071114.

\section{References}

[1] Chang R K and Campillo A J 1996 Optical Processes in Microcavities (Singapore: World Scientific)

[2] Gmachl C, Capasso F, Narimanov E E, Nöckel J U, Stone A D, Faist J, Sivco D L and Cho A Y 1998 Science $\mathbf{2 8 0} 1566$

[3] Schwefel H G L, Rex N B, Tureci H E, Chang R K, Stone A D and Zyss J 2003 arXiv:physics/0308001 v1

[4] Chern G D, Tureci H E, Stone A D, Chang R K, Kneissl M and Johnson N M 2003 Appl. Phys. Lett. 831710

[5] Slusher R E, Levi A F J, Mohideen U, McCall S L, Pearton S J and Logan R A 1993 Appl. Phys. Lett 631310

[6] Zhang J P, Chu D Y, Wu S L, Ho S T, Bi W G, Tu C Y and Tiberio R C 1995 Phys. Rev. Lett. 752678

[7] Mitchell K A, Handley J P, Tighe Flower B A and Delos J B 2004 Phys. Rev. Lett. 92073001

[8] Mitchell K A, Handley J P, Tighe B, Delos J B and Knudson S K 2003 Chaos 13880

[9] Mitchell K A, Handley J P, Delos J B and Knudson S K 2003 Chaos 13892

[10] Bohigas O, Giannoni M Jand Schmit C 1984 Phys. Rev. Lett. 521

[11] Heller E J 1984 Phys. Rev. Lett. 531515

[12] Kaplan L 1998 Phys. Rev. Lett. 802582

[13] Kaplan L and Heller E J 1998 Ann. Phys. 264171

[14] Kaplan L 1999 Nonlinearity 12 R1

[15] Creagh S C, Lee S-Y and Whelan N D 2002 Ann. Phys. 295194

[16] Lee S-Y and Creagh S C 2003 Ann. Phys. 307392

[17] Bogomolny E B 1988 Physica D 31169

[18] Berry M V 1989 Proc. Roy. Soc. A 243219

[19] Sridhar S 1991 Phys. Rev. Lett. 67785

[20] Stein J and Stöckman H-J 1992 Phys. Rev. Lett. 682867

[21] Fromhold T M, Wilkinson P B, Sheard F W, Eaves L, Miao J and Edwards G 1995 Phys. Rev. Lett. 751142

[22] Wilkinson P B, Fromhold T M, Eaves L, Sheard F W, Miura N and Takamasu T 1996 Nature 380608

[23] Kudrolli A, Abraham M C and Gollub J P 2001 Phys. Rev. E 63026208

[24] Stöckman H-J and Stein J 1990 Phys. Rev. Lett. 642215

[25] Sridhar S and Heller E J 1992 Phys. Rev. A 46 R1728 
[26] Lee S-B, Lee J-H, Chang J-S, Moon H-J, Kim S-W and An K 2002 Phys. Rev. Lett. 88033903

[27] C. Gmachl, E. E. Narimanov, F. Capasso, J. N. Ballargeon, and A. Y. Cho 2002 Opt. Lett. 27 824

[28] Rex N B, Tureci H E, Schwefel H G L, Chang R K and Stone A D 2002 Phys. Rev. Lett. 88 094102

[29] Harayama T, Fukushima T, Davis P, Vaccaro P O, Miyasaka T, Nishimura T and Aida T 2003 Phys. Rev. E 67, 015207(R)

[30] Lee S-Y, Ryu J-W, Kwon T-Y, Rim S, and Kim C-M 2005 Phys. Rev. A 72 061801(R)

[31] Lee S-Y, Rim S, Ryu J-W, Kwon T-Y, Choi M and Kim C-M 2004 Phys. Rev. Lett. 93164102

[32] Ryu J-W, Lee S-Y, Kim C-M and Park Y-J 2006 Phys. Rev. E 73036207

[33] Hawkes J and Latimer I 1995 Lasers; Theory and Practice (Prentice Hall)

[34] Fendrik A J, Rivas A M F and Sánchez M J 1994 Phys. Rev. E 501948

[35] Chirikov B V and Shepelyansky D L 1984 Physica D 13395

[36] Lai Y C, Ding M, Grebogi C and Blümel R 1992 Phys. Rev. A 464661

[37] Weiss M, Hufnagel L and Ketzmerick R 2003 Phys. Rev. E 67046209

[38] Wiersig J 2003 J. Opt. A: Pure Appl. Opt. 553

[39] Cohen D, Lepore N and Heller E J 2004 J. Phys. A 372139

[40] Baltes H P and Hilf E R 1976 Spectra of Finite Systems (Mannheim: B. I. Wissenschaftsverlag)

[41] Lee S-Y, Kurdoglyan M S, Rim S and Kim C-M 2004 Phys. Rev. A 70023809

[42] Schwefel H G L, private commucation.

[43] Rim S et al. (in preparation)

[44] Hentschel M, Schomerus H and Schubert R 2003 Europhys. Lett. 62636

[45] H. Schomerus H and Hentschel M 2006 Phys. Rev. Lett. 96243903

[46] Goos F and Hänchen H 1947 Ann. Phys. (Leipzig) 1333

[47] Hentschel M and Schomerus H 2002 Phys. Rev. E 65 045603(R)

[48] Tureci H E and Stone A D 2002 Opt. Lett. 277

[49] Carlo G G, Vergini E G and Lustemberg P 2002 J. Phys. A: Math. Gen. 357965 